\title{
Impact of secondary particles on microdistribution of deposited dose in biological tissue in the presence of gold and gadolinium nanoparticles under photon beam irradiation*
}

\author{
Ivan A. Konobeev ${ }^{1}$, Yurij A. Kurachenko ${ }^{2}$, Igor' N. Sheino ${ }^{1}$ \\ 1 SRC-Burnasyan Federal Medical Biophysical Center of Federal Medical Biological Agency, 46 Zhivopisnaya str., Moscow, 123182 Russia \\ 2 Russian Institute of Radiology and Agroecology, 109 km Kievskoye shosse, Obninsk, Kaluga reg., 249032 Russia \\ Corresponding author: Ivan A. Konobeev (beo0@mail.ru)
}

Academic editor: Yury Korovin • Received 27 February 2019 • Accepted 27 April 2019 • Published 17 May 2019

Citation: Konobeev IA, Kurachenko YA, Sheino IN (2019) Impact of secondary particles on microdistribution of deposited dose in biological tissue in the presence of gold and gadolinium nanoparticles under photon beam irradiation. Nuclear Energy and Technology 5(2): 109-116. https://doi.org/10.3897/nucet.5.35798

\begin{abstract}
It is experimentally proven that nanoparticles of high- $\mathrm{Z}$ materials can be used as radiosensitizers for photon beam therapy. In the authors' opinion, data available as of today on the impact of secondary particles (electrons, photons and positrons generated in biological tissue by penetrating beam of primary photons) on the distribution of deposited dose during photon beam therapy in the presence of nanoparticles, are insufficient. Investigation of this impact constituted the main goal of this work.

Two-stage simulation was performed using Geant4 platform. During the first stage a layer of biological tissue (water) was irradiated by monoenergetic photon sources with energies ranging from $10 \mathrm{keV}$ to $6 \mathrm{MeV}$. As the result of this modeling spectra of electrons, photons and positrons were obtained at the depth of $5 \mathrm{~cm}$. During the second stage the obtained photon spectra were used to irradiate gold, gadolinium and water nanoparticles. Radial distributions of energy deposited around nanoparticles were obtained as the result of this modeling.

Radial DEF (Dose Enhancement Factor) values around nanoparticles of gold and gadolinium positioned in water at the depth of $5 \mathrm{~cm}$ were obtained after processing the collected data. Contributions from primary photons and secondary particles (electrons, photons and positrons generated in the layer of water with 5-cm thickness by the penetrating beam of primary photons) in the additional dose deposited around the nanoparticles were calculated as well.

It was demonstrated that layer of biological tissue placed between the source of photons and nanoparticles considerably changes the initial spectrum of photons and this change is significant in the analysis of mechanism of radiosensitization of biological tissues by nanoparticles for all energies of photon sources (up to $6 \mathrm{MeV}$ ).

It was established that interaction of electrons and positrons with nanoparticles does not lead to significant increase of additional dose in the vicinity of their surfaces and can be most likely excluded from consideration in the analysis of radiosensitization mechanism of nanoparticles.
\end{abstract}

\section{Keywords}

Nanoparticles of gold and gadolinium; deposited dose; photon beam therapy; Geant4; Monte Carlo simulation

* Russian text published: Izvestiya vuzov. Yadernaya Energetika (ISSN 0204-3327), 2019, n. 1, pp. 155-165.

Copyright Konobeev IA et al. This is an open access article distributed under the terms of the Creative Commons Attribution License (CC-BY 4.0), which permits unrestricted use, distribution, and reproduction in any medium, provided the original author and source are credited. 


\section{Introduction}

Methods for physical aiming of radiation on the tumor, such as three-dimensional conformal radiation therapy (3D CRT), intensity-modulated radiotherapy (IMRT), image guided radiation therapy (IGRT), stereotactic radiation surgery (SRT) ensuring maximum precision of radiation dose rendering to tumor target have reached the limits of their further refinement (Van Dyk 2005). It is considered that significant progress in the efficiency of radiation therapy can be reached as the result of application of radiation sensitizers (Connell and Hellman 2009, Lehnert 2015). Radiation sensitizers are, as a rule, the chemical or pharmacological agents augmenting necrocytosis under irradiation when these agents are present in biological tissue. Important feature of radiation sensitizers is their differentiated behavior with respect to normal tissues and tumors, i.e. they must increase sensitivity of tumors higher than that of healthy tissues. Different approaches are applied to achieve this including the most recent developments of molecular target preparations (Bergs et al. 2015).

One of the options of radiosensitization is the introduction in the biological medium of elements with significantly higher radiation absorption cross-section than that of the biological tissue per se. Emerging secondary short-range radiation localizes energy absorption in the vicinity of these elements and affects only adjacent biological structures.

Main principles of binary radiation therapy technologies such as the neutron capture therapy (NCT) or photon capture therapy (PCT) refer to the methods of radiation sensitization. In PCT technology additional energy yield under irradiation of biological tissue with photons is caused by the emission of photoelectrons and accompanying Auger-cascade from atoms of "heavy" elements (high- $Z$ elements): ${ }_{53} \mathrm{I},{ }_{64} \mathrm{Gd},{ }_{78} \mathrm{Pt},{ }_{79} \mathrm{Au}$ and other elements included in the compositions of a number of preparations (Sheino 2006). That is from where the concept of photon capture therapy emerged (Khokhlov et al. 2006). Gadolinium $(Z=64)$, platinum $(Z=78)$ and gold $(Z=79)$ are the most widely used elements in the investigation of radiation sensitization.

The most widely spread approach to the explanation of radiation sensitization of biological tissue under photon radiation is the local increase of deposited dose in the biological medium due to the presence in the medium of elements with high atomic number (Kwatra et al. 2013).

In vitro and in vivo experiments demonstrated significant effects of radiation sensitization by nanoparticles of gold for different cellular lines with application of X-ray beams within kilovolt range (Chithrani et al. 2010, Liu et al. 2010, Jain et al. 2011, Hainfeld et al. 2004). At the same time a number of experimental studies showed potential possibility of application of gold nanoparticles for radiation sensitization using clinical megavolt photon beams of linear accelerators (Chithrani et al. 2010, Liu et al. 2010, Jain et al. 2011, Berbeco et al. 2012, Butterworth et al. 2012, Tsiamas et al. 2013, Detappe et al. 2015). These and other studies demonstrated radiation sensitization for different cellular lines for concentrations of "heavy" elements which were much lower than those needed for noticeable increase of total deposited dose in the cells.

The reason of discrepancy between calculated values of increase of deposited dose and observable biological effect of necrocytosis is the significant heterogeneity of the dose field emerging in the case of manifested localization of energy absorption in the direct vicinity from "heavy" elements which may reach extremely high values (Sheino et al. 2014) (Fig. 1).

Such significant heterogeneities of dose can become the cause of a whole series of biochemical processes,
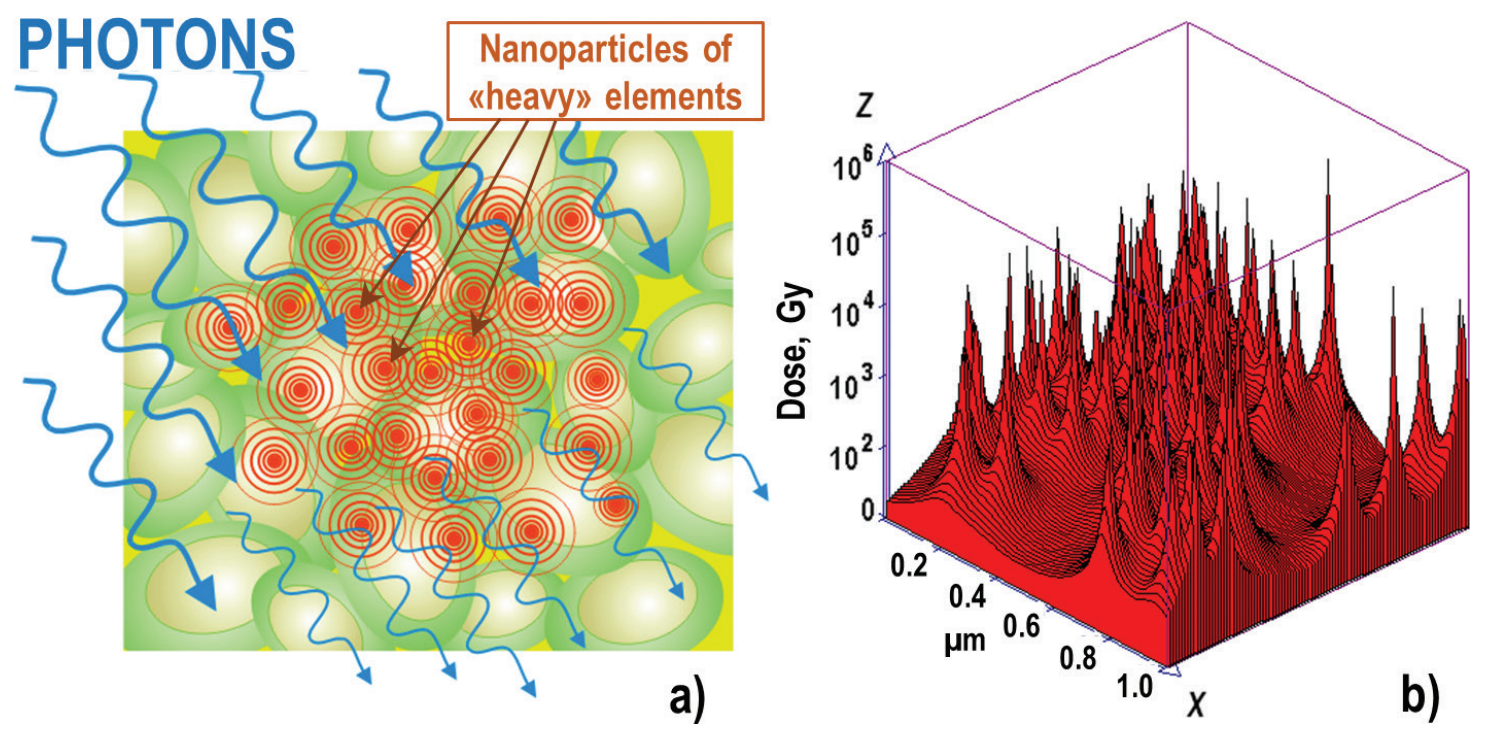

Figure 1. Schematic illustration of generation of heterogenous micro-distribution of deposited dose after activation of "heavy" elements in biological tissue (a) and corresponding diagram of deposited dose (b). 
including high generation of deleterious and hydroxyl radicals and other ensuing processes of complex biological damage (Detappe et al. 2015, McMahon et al. 2011).

The heterogeneity in question appears under the effects on heavy elements of both photons emitted by the source, and of secondary particles generated in the biological medium (photons, electrons and positrons).

As of today, available data on the effects of secondary particles on dose heterogeneity in the vicinity of heavy elements are insufficient. Effects of electrons are often disregarded without indicating the uncertainty introduced by such approximation.

The objective of the present study is the investigation of distributions of deposited dose in biological tissue in the presence of nanoparticles of gold and gadolinium depending on the photon source energy and effects of secondary particles generated in the biological medium on these distributions.

\section{Terminology}

All photons are divided in the paper into two groups: "primary" and "secondary" photons. Photons are considered to be "primary" if their energy is equal to the energy of photons emitted from monoenergetic source. In particular, photons undergoing Raleigh scattering remain to be "primary". Photons are considered to be "secondary" if their energy is less than the energy of photons emitted from the source. In particular, photons that underwent Compton scattering or photons emitted as the result of annihilation are attributed to the group of "secondary" photons.

\section{Materials and methods}

One nanoparticle positioned at the depth of $5 \mathrm{~cm}$ from flat monoenergetic photon source was simulated for investigating distribution of additional dose associated with presence of nanoparticles in the water (as a tissue-equivalent material). The geometry in question is a simplified model of small-size tumor located at the depth of $5 \mathrm{~cm}$ in human body and containing nanoparticles. The problem had to be solved in two stages because of the disproportion between the dimensions of the photon beam and the nanoparticle size. Each stage represented separate calculation using Geant4 platform of version 10.1 (Agostinelli et al. 2003). Homogenous photon beam penetrating the layer of water was simulated during the first stage. The main results obtained were particle spectra generated in water at the depth of $5 \mathrm{~cm}$. The second stage consisted of simulation of single nanoparticle irradiated by the source (equivalent source) positioned immediately adjacent to the nanoparticle and ascribed the spectrum obtained during the first stage.

\section{First stage}

The main objective of the first stage was obtaining spectra of electrons, photons and positrons generated in water after irradiation by monoenergetic photon source with energy within the range from $10^{\circ} \mathrm{keV}$ to $6^{\circ} \mathrm{MeV}$.

The following problem was simulated. Flat monoenergetic homogenous photon source with $10-\mathrm{cm}$ diameter is located in the center of water cylinder base with $20-\mathrm{cm}$ height and $20-\mathrm{cm}$ diameter. Photons escape the source at right angle to its surface. The cylinder contains detector positioned at the distance of $5 \mathrm{~cm}$ from its base and registering the spectra of particles passing through it. The detector is a cylinder of water with thickness equal to $1 \mu \mathrm{m}$ and diameter equal to $20 \mathrm{~cm}$ (Fig. 2). Spectra of electrons, photons and positrons passing through the detector were stored in the process of simulation. The height of the bigger cylinder $(20 \mathrm{~cm})$ was chosen to be significantly larger than it is necessary for determining the above indicated spectra for obtaining additional results not provided in the present paper.

Livermore (in a larger cylinder) and DNA (in the detector) physical models (Geant4, Karamitros et al. 2014, Incerti et al. 2010) were used for describing propagation of electrons. Threshold of secondary particle generation in the larger cylinder was equal to $1 \mathrm{keV}$. Propagation of electrons in the detector was simulated down to the energy equal to $5.1 \mathrm{eV}$.

Spectra of electrons, photons and positrons were stored with pitch equal to $1^{\circ} \mathrm{keV}$. Therefore, spectra obtained for source with maximum energy of $6 \mathrm{MeV}$ contained 6000 points each. Spectrum of electrons with energies up to $1 \mathrm{keV}$ was stored separately with pitch equal to $1 \mathrm{eV}$ and contained 1000 points. Spectra of only those particles which were flying at angles $[0 ; \pi / 2)$ against the direction of flight of photons escaping the source were stored during simulation.

\section{Second stage}

The main objective of this stage was the determination of radial distributions of absorbed energy around nanoparticles irradiated with equivalent source of photons, electrons and positrons with spectra obtained during the first stage of simulation.

The following problem was simulated. Single nanoparticle (homogenous spherical ball with diameter equal to 2 $\mathrm{nm}$ and $30 \mathrm{~nm}$ consisting of gold, gadolinium and water) was irradiated by circular source with diameter equal to the diameter of the nanoparticle positioned directly adjacent to it (see Fig. 2). Spectra of the source were taken from the results of the first stage of simulation. Spectra of the following two types were used: average spectrum along the central part of the detector (over the circular disc at the center of the detector with radius equal to the radius of the source from the first stage of simulation) and spectrum averaged over the annular part of the detector (annulus located directly adjacent to the central part of the detector with inner radius equal to the radius of the source and outer radius exceeding the inner radius by 1 $\mathrm{cm}$ ). These spectra were regarded as the spectra "inside the beam" and "outside the beam", respectively. The area 

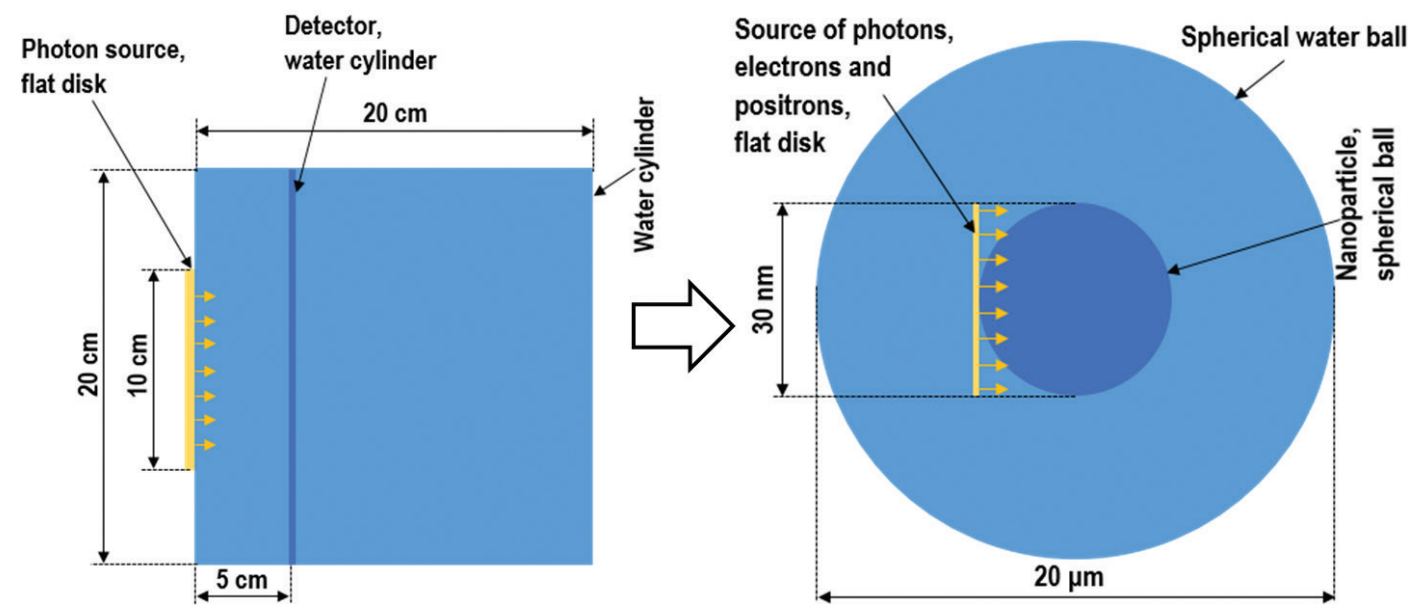

Figure 2. Calculation geometries for the first (left) and second (right) stages of simulation. Nanoparticles with diameters equal to 2 $\mathrm{nm}$ and $30 \mathrm{~nm}$ were simulated during the second stage.

"outside the beam" was selected in such a way as to have the particle fluence inside it lower by one order of magnitude than that "inside the beam" (see Fig. 3). Angular distribution of particles was neglected and simulation was performed for all particles as if they were emitted at the angle equal to 0 degrees against the source axis.

Energy absorbed in spherical layers with $1 \mathrm{~nm}$ thickness surrounding nanoparticle up to the distance of 10 $\mu \mathrm{m}$ from the center of the nanoparticle was stored during simulation. Absorbed energy was stored separately for the following four types of particles emitted from the source: electrons, "primary" photons (photons with energy equal to the energy of primary photons during the first stage of simulation), "secondary" photons (photons with energies smaller than the energy of primary photons during the first stage of simulation) and positrons.

Livermore (inside nanoparticle) and DNA (inside the spherical water ball) physical models were used for describing propagation of electrons. Threshold of secondary particle generation in the nanoparticle was equal to $10 \mathrm{eV}$. Propagation of electrons in the water ball was simulated down to the energy equal to $5.1 \mathrm{eV}$. Propagation of ions and radicals was not taken into account.

Radial DEF (Dose Enhancement Factor, i.e. the ratio of dose in the presence of nanoparticle of gold or gadolinium to the dose value in the absence of nanoparticle) values and contributions from electrons, primary photons, secondary photons and positrons in the additional deposited dose within the area from the surface to $100 \mathrm{~nm}$ from the center of the nanoparticle (area of peak radial dose, see Fig. 5) were calculated according to the radial distributions of absorbed energy around nanoparticles. Calculations of $D E F$ values within each spherical layer were performed according to the following formula:

$$
D E F_{X N P}=1+\left(D_{X N P}-D_{W N P}\right) / D^{*}
$$

where $D_{X N P}$ is the calculated average dose in the spherical layer surrounding the nanoparticle consisting of materi- al $X$ (gold or gadolinium); $D_{W N P}$ is the calculated average dose in the same spherical layer surrounding the nanoparticle consisting of water and irradiated with the same particle beam; $D^{*}$ is the average dose in the water in the absence of nanoparticles consisting of material $X$ calculated as the average calculated dose inside nanoparticle consisting of water. Each dose value was calculated as the ratio of energy absorbed inside the volume to its mass.

Thus obtained $D E F$ values are close to those which are expected to be observed in experiment.

Contribution of each type of particles in the additional dose was calculated as the difference between deposited doses from the given type of particles when they irradiate nanoparticle made of agent material and nanoparticle consisting of water.

\section{Results and discussion}

\section{First stage}

Dependences of fluences of electrons, photons and positrons emitted at the depth of $5 \mathrm{~cm}$ on the energy of primary photons for monoenergetic source are presented in Figure 3. Fluence "inside the beam" is the fluence of particles passing through the central part of the detector (see Fig.2) with diameter equal to the diameter of the source $(10 \mathrm{~cm})$. Fluence "outside the beam" is the fluence of particles passing through the detector annular layer with inner diameter equal to the diameter of the source and outer diameter with value exceeding the inner diameter by $1 \mathrm{~cm}$.

At the energy of the source equal to $10 \mathrm{keV}$ fluences for all particles at the depth of $5 \mathrm{~cm}$ are equal to zero. Corridors of uncertainties for photons inside the beam are very narrow and, therefore, they are indistinguishable in the plots.

Spectra of photons and electrons emitted in the water at the depth of $5 \mathrm{~cm}$ from the monoenergetic photon sources 
with energies equal to $300 \mathrm{keV}, 1000 \mathrm{keV}$ and $6000 \mathrm{keV}$ are presented in Figure 4. Jumps in the plots attributed to statistical uncertainty of calculations were smoothed (averaged) for easier visual comprehension.

As it has been described above in the section "Materials and Methods" spectra were stored with pitch along the energy axis equal to $1 \mathrm{keV}$. Therefore, fluence of particles with energy $[E ; E+1) \mathrm{keV}$, where $E$ is the positive integer value corresponds to the point with energy value equal to $(E+0.5)^{\circ} \mathrm{keV}$.

A large number of electrons "inside the beam" with energies within $1 \mathrm{keV}$ (about 4.1 and $0.6 \%$ of the total number of electrons "inside the beam" for 300, 1000 and 6000 $\mathrm{keV}$, respectively) are also present in the spectra. Spectra in the region have pronounced peak within the range of $8-20 \mathrm{eV}$. In the process of irradiation of nanoparticle these electrons reduce additional deposited dose associated with presence of the nanoparticle by being efficiently absorbed inside the nanoparticle.

As it is clear from Fig. 4a, low energy component appears in the photon spectrum during penetration of monoenergetic photon beam through the layer of water. At the energies of primary photons exceeding two electron rest energies $(1022 \mathrm{keV})$ large number of photons with energy of $511 \mathrm{keV}$ (annihilation peak) are formed in the spectrum.

\section{Second stage}

Radial distributions of $D E F$ in the layers surrounding nanoparticles positioned in the water at the depth of $5 \mathrm{~cm}$ and irradiated with monoenergetic photon sources (from the first stage) are presented in Figure 5.

Similar to the cross-section of photon interaction with agent material, additional dose decreases with increased photon energy and has a jump corresponding to the penetration through K-shell of the agent material (energy of K-shell amounts to approximately $80.7 \mathrm{keV}$ for gold and to $50.2^{\circ} \mathrm{keV}$ for gadolinium). Within the range from $600 \mathrm{keV}$ to $6000^{\circ} \mathrm{keV}$ "inside-the-beam" $D E F$ curves are very close to each other and are not discernible within the limits of calculation uncertainty.

It has to be noted that while radial $D E F$ in Fig. 5 give certain understanding about efficiency of different photon energies for radiation sensitization, they cannot be used for quantitative description of the biological effect. The most advanced models for determining the biological effect (Cunha et al. 2017) require analysis of separate particle tracks, while the data in Fig. 5 are averaged over the volumes of spherical layers and the number of primary photons.

Histograms of contributions from each type of particles escaping from the equivalent source (electrons, primary photons, secondary photons and positrons) in the additional deposited dose within the area from the surface to $100 \mathrm{~nm}$ from center of the nanoparticles with 30 -nm diameter positioned inside the beam, are presented in Figure 6.

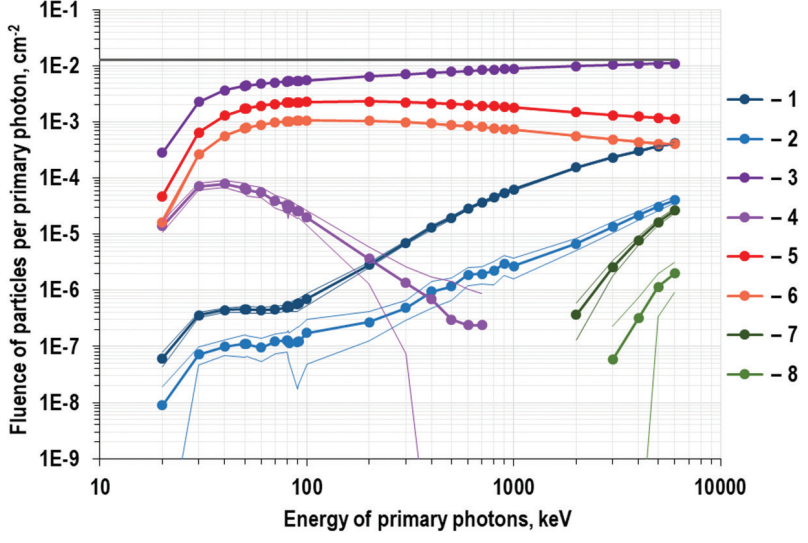

Figure 3. Fluences of particles per primary photon generated at the depth of $5 \mathrm{~cm}$ from the monoenergetic photon source inside and outside of the original beam: 1 - electrons inside the beam; 2 - electrons outside the beam; 3 - primary photons inside the beam; 4 - primary photons outside the beam; 5 - secondary photons inside the beam; 6 - secondary photons outside the beam; 7 - positrons inside the beam; 8 - positrons outside the beam. Thin lines indicate the corridors of statistical uncertainties of calculations; upper straight line corresponds to the initial fluence of the source (photon fluence at the depth of $0 \mathrm{~cm}$ ).
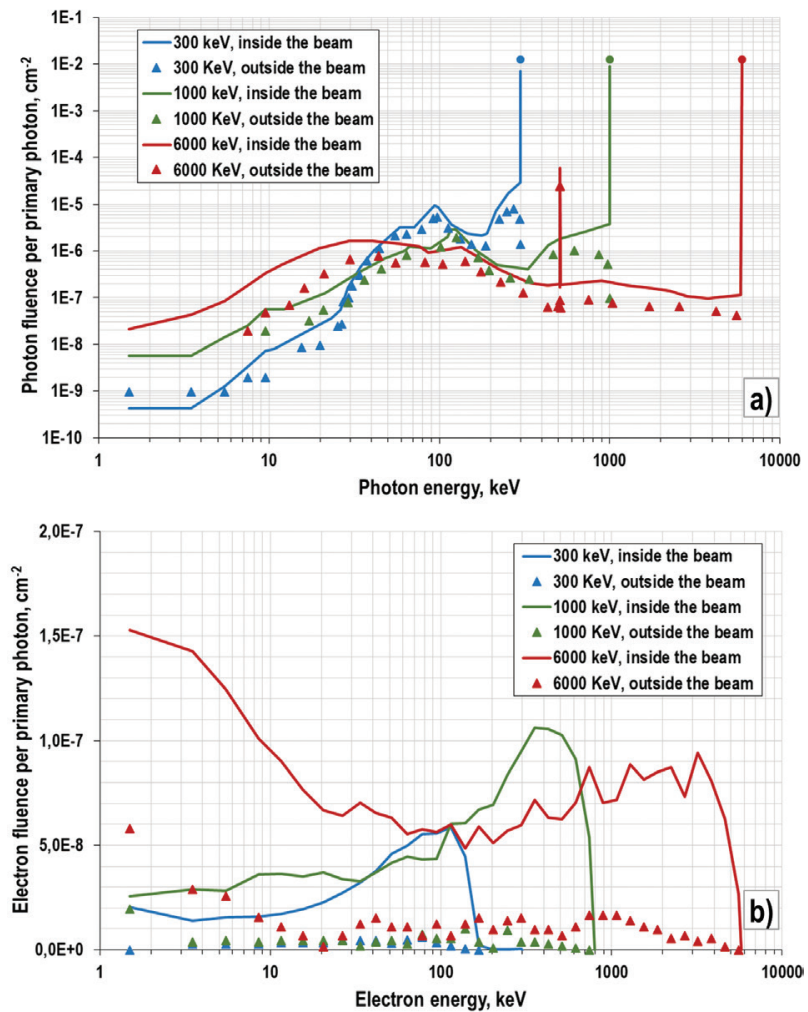

Figure 4. Spectra of photons (a) and electrons (b) emerging in the water at the depth of $5 \mathrm{~cm}$ from the monoenergetic photon source. Black circles in Figure (a) indicate initial fluences of the sources (photon fluences at the depth of $0 \mathrm{~cm}$ ).

Thus, photon spectrum changes significantly when photon beam penetrates through the water (Fig. 4a). Photons with lower energies which ionize nanoparticles 

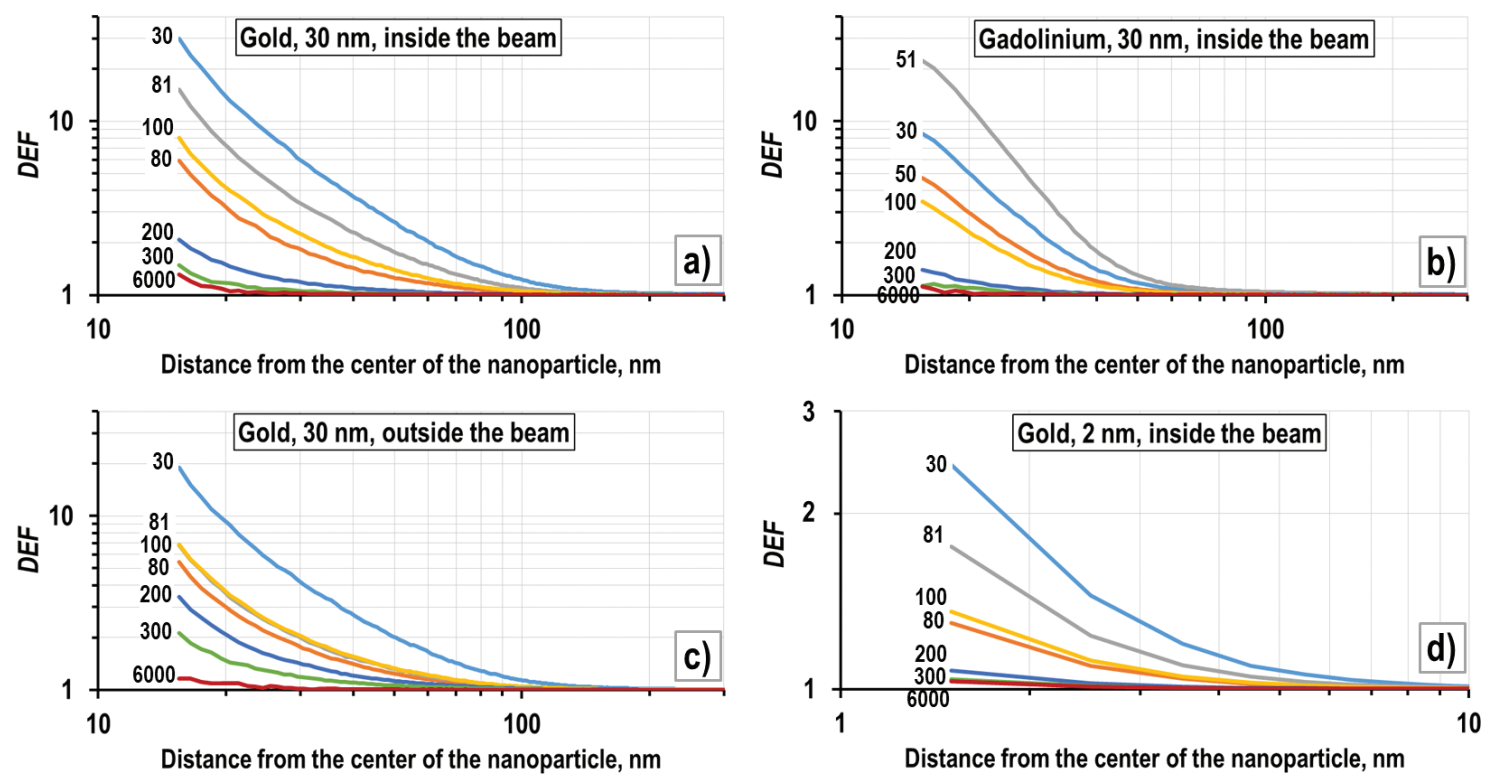

Figure 5. Radial $D E F$ for nanoparticles: a) - Au $\phi 30 \mathrm{~nm}$, inside the beam; b) - Gd $\phi 30 \mathrm{~nm}$, inside the beam; c) - Au $\phi 30 \mathrm{~nm}$, outside the beam; d) - Au $\phi 2 \mathrm{~nm}$, inside the beam. Figures near the curves indicate energies of primary photons in keV. Curves for $81 \mathrm{keV}$ and $100^{\circ} \mathrm{keV}$ in Fig. c) practically coincide.
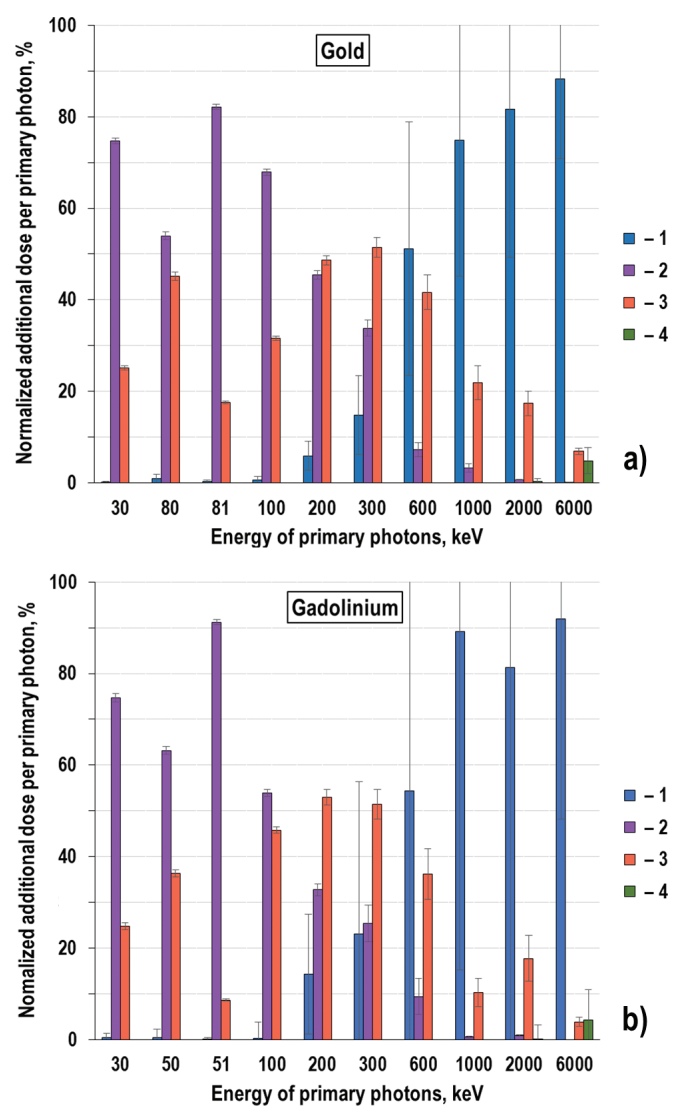

Figure 6. Contributions in the additional dose within the area from the surface to $100 \mathrm{~nm}$ from the center of nanoparticles of gold and gadolinium with $\phi 30 \mathrm{~nm}$ positioned inside the beam from electrons, primary photons, secondary photons and positrons: 1 - electrons; 2 - primary photons; 3 - secondary photons; 4 - positrons. Statistical uncertainties are indicated with bars. more intensively appear in the spectrum. Contribution of secondary photons in the additional dose in the vicinity of nanoparticle surface appears to be significant for all examined energies of the primary photon source (see Fig. 6). Comparing the ratio of fluences of primary and secondary photons in Fig. 3 with the ratio of contributions of primary and secondary photons in the additional dose in Fig. 6, conclusion can be drawn that for all energies represented in Fig. 6, with exception to $51^{\circ} \mathrm{keV}$ for gadolinium and $81^{\circ} \mathrm{keV}$ for gold, secondary photons are more efficient than primary photons as pertains to the increase of additional dose near the surfaces of nanoparticles. Thus, layer of water positioned between the source and the area containing nanoparticles changes the spectrum making it more efficient (with regard to the increase of additional dose near the nanoparticle surfaces) for irradiating nanoparticles for all energies of the source within the range from $30 \mathrm{keV}$ to $6000^{\circ} \mathrm{keV}$ with exception of source energies a little higher than $\mathrm{K}$-shell for the material of nanoparticles.

Conclusion can be drawn by comparing Figures 5 and 6 that ionization of nanoparticles by electrons and positrons cannot result in the significant additional dose in the vicinity of surfaces of nanoparticles - no significant additional dose is observed for energies at which contribution of electrons and positrons is substantial.

Let us note that while water layer increases efficiency of the photon spectrum per se in its presence $D E F$ around nanoparticles will be significantly lower than in its absence because of considerably higher flux of electrons which cannot efficiently ionize nanoparticle and, therefore, they will be leveling the ratio of values of doses in the presence of nanoparticle and in its. 


\section{Conclusion}

Sharp jumps of deposited dose in the vicinity of surfaces of nanoparticles emerge under irradiation of biological tissue with photons with energies up to $6 \mathrm{MeV}$. This is explained, in the first place, by photoelectric absorption of photons in nanoparticles with subsequent emission of short-range electrons.

Irradiation with photons of tissue equivalent phantom results in the emergence of low-energy components in the photon spectrum inside the tissue and in the appearance of fluxes of electrons and positrons. It was established that such variation of photon spectrum is significant in the analysis of the mechanism of radiation sensitization of biological tissue by nanoparticles for any energies of the photon source (up to $6^{\circ} \mathrm{MeV}$ ).

It was demonstrated that interaction of electrons and positrons with nanoparticles does not result in significant growth of additional dose in the vicinity of surfaces of nanoparticles and, most likely, can be excluded from further examination in the analysis of the mechanism of radiation sensitization of biological tissue by nanoparticles.

For quantitative description of radiation sensitization of tissues by nanoparticles it is necessary to take into account a number of biochemical processes, including high rate of generation of deleterious hydroxyl radicals and other subsequent processes of complex biological damages.

\section{References}

- Agostinelli S, Allison J, Amako K, Apostolakis J, Araujo H, Arce P, Asai M, Axen D, Banerjee S, Barrand G, Behner F, Bellagamba L, Boudreau J, Broglia L, Brunengo A, Burkhardt H, Chauvie S, Chuma J, Zschiesche D (2003) Geant4 - a simulation toolkit. Nuclear Instruments and Methods in Physics Research 506(3): 250-303. https://doi.org/10.1016/S0168-9002(03)01368-8

- Berbeco RI, Korideck H, Ngwa W, Kumar R, Patel J, Sridhar S, Johnson S, Price BD, Kimmelman A, Makrigiorgos GM (2012) DNA damage enhancement from gold nanoparticles for clinical MV photon beams. Radiation Research 178(6): 604-608. https://doi. org/10.1667/RR3001.1

- Bergs JW, Wacker MG, Hehlgans S, Piiper A, Multhoff G, Rödel C, Rödel F (2015) The role of recent nanotechnology in enhancing the efficacy of radiation therapy. Biochimica et Biophysica Acta 1856(1): 130-143. https://doi.org/10.1016/j.bbcan.2015.06.008

- Butterworth KT, McMahon SJ, Currell FJ, Prise KM (2012) Physical basis and biological mechanisms of gold nanoparticle radiosensitization. Nanoscale 4(16): 4830-4838. https://doi.org/10.1039/ c2nr31227a

- Chithrani DB, Jelveh S, Jalali F, van Prooijen M, Allen C, Bristow RG, Hill RP, Jaffray DA (2010) Gold nanoparticles as radiation sensitizers in cancer therapy. Radiation Research 173(6): 719-728. https://doi.org/10.1667/RR1984.1

- Connell PP, Hellman S (2009) Advances in radiotherapy and implications for the next century: a historical perspective. Cancer Research 69(2): 383-392. https://doi.org/10.1158/0008-5472.CAN-07-6871

- Cunha M, Monini C, Testa E, Beuve M (2017) NanOx, a new model to predict cell survival in the context of particle therapy. Physics in Medicine and Biology 62(4): 1248-1268. https://doi org/10.1088/1361-6560/aa54c9

- Detappe A, Kunjachan S, Rottmann J, Robar J, Tsiamas P, Korideck H, Tillement O, Berbeco R (2015) AGuIX nanoparticles as a promising platform for image guided radiation therapy. Cancer Nanotechnology 6(1): 4. https://doi.org/10.1186/s12645-015-0012-3

- Geant4 physics reference manual (2018) Geant4 physics reference manual. http://geant4-userdoc.web.cern.ch/geant4-userdoc/UsersGuides/PhysicsReferenceManual/fo/PhysicsReferenceManual.pdf [Accessed Nov 8, 2018] Hainfeld JF, Slatkin DN, Smilowitz HM (2004) The use of gold nanoparticles to enhance radiotherapy in mice. Physics in medicine and biology, 49 (N309-N315). https://doi.org/10.1088/00319155/49/18/N03

- Incerti S, Ivanchenko A, Karamitros M, Mantero A, Moretto P, Tran HN, Mascialino B, Champion C, Ivanchenko VN, Bernal MA, Francis Z, Villagrasa C, Baldacchino G, Guèye P, Capra R, Nieminen P, Zacharatou C (2010) Comparison of Geant4 very low energy cross section models with experimental data in water. Medical Physics 37(9): 4692-4708. https://doi.org/10.1118/1.3476457

- Jain S, Coulter JA, Hounsell AR, Butterworth KT, McMahon SJ, Hyland WB, Muir MF, Dickson GR, Prise KM, Currell FJ, O’Sullivan JM, Hirst DG (2011) Cell-specific radiosensitization by gold nanoparticles at megavoltage radiation energies. International Journal of Radiation Oncology Biology Physics 79(2): 531-539. https:// doi.org/10.1016/j.ijrobp.2010.08.044

- Karamitros M, Luan S, Bernal MA, Allison J, Baldacchino G, Davidkova M, Francis $Z$, Friedland W, Ivantchenko V, Ivantchenko A, Mantero A, Nieminen P, Santin G, Tran HN, Stepan V, Incerti $\mathrm{S}$ (2014) Diffusion-controlled reactions modeling in Geant4-DNA. Journal of Computational Physics 274(1): 841-882. https://doi. org/10.1016/j.jcp.2014.06.011

- Khokhlov VF, Sheino IN, Kulakov VN, Mitin VN, Nasonova TA, Dobrynina OA (2006) The method of photon-capture therapy of tumors. Patent RF №2270045. [In Russian]

- Kwatra D, Venugopal A, Anant S (2013) Nanoparticles in radiation therapy: a summary of various approaches to enhance radiosensitization in cancer. Translational Cancer Research 2(4): 330-342.

- Lehnert S (2015) Radiosensitizers and Radiochemotherapy in the Treatment of Cancer. CRC Press, Boca Raton, 548 pp.

- Liu C-J, Wang C-H, Chen S-T, Chen HH, Leng WH, Chien CC, Wang CL, Kempson IM, Hwu Y, Lai TC, Hsiao M, Yang CS, Chen YJ, Margaritondo G (2010) Enhancement of cell radiation sensitivity by pegylated gold nanoparticles. Physics in Medicine and Biology 55(4): 931-945. https://doi.org/10.1088/0031-9155/55/4/002

- McMahon SJ, Hyland WB, Muir MF, Coulter JA, Jain S, Butterworth KT, Schettino G, Dickson GR, Hounsell AR, O'Sullivan JM, Prise KM, Hirst DG, Currell FJ (2011) Biological consequences of 
nanoscale energy deposition near irradiated heavy atom nanoparticles. Scientific Reports 1: no. 18. https://doi.org/10.1038/srep00018

- Sheino IN (2006) Dose-supplementary therapy of malignant tumors. Advances in Neutron Capture Therapy. In: Nakagawa Y, Kobayashi T, Fukuda H (Eds) Proceedings of ICNCT-12. Takamatsu, 531-534.

- Sheino IN, Konobeev IA, Izhevskiy PV, Fedotov YuA (2014) Estimation of relative biological effectiveness of heterogeneous microdistribution of the deposited dose in binary technologies of radiation therapy. Proc. of the VI Scient. Conf. "Medical physics and innovations in medicine”. Troitsk, 223-226. [In Russian]
- Tsiamas P, Liu B, Cifter F, Ngwa WF, Berbeco RI, Kappas C, Theodorou K, Marcus K, Makrigiorgos MG, Sajo E, Zygmanski P (2013) Impact of beam quality on megavoltage radiotherapy treatment techniques utilizing gold nanoparticles for dose enhancement. Physics in Medicine and Biology 58(3): 451-464. https://doi. org/10.1088/0031-9155/58/3/451

- Van Dyk J (2005) Advances in modern radiation therapy. In: Van Dyk J (Ed.) The Modern Technology of Radiation Oncology. Medical Physics Pub Corp, 1-31. 